Cahiers $d u$ MONDE RUSSE

\section{Cahiers du monde russe}

Russie - Empire russe - Union soviétique et États indépendants

$49 / 4 \mid 2008$

Destins individuels et terreur. Jeunesse dans la société post-stalinienne

\title{
Ray Brandon, Wendy Lower, eds, The Shoah in Ukraine
}

Nathalie Moine

\section{OpenEdition}

Journals

Édition électronique

URL : https://journals.openedition.org/monderusse/7009

DOI : 10.4000/monderusse. 7009

ISSN : $1777-5388$

Éditeur

Éditions de l'EHESS

Édition imprimée

Date de publication : 28 décembre 2008

Pagination : 833-838

ISBN : 978-2-7132-2197-2

ISSN : $1252-6576$

Référence électronique

Nathalie Moine, "Ray Brandon, Wendy Lower, eds, The Shoah in Ukraine », Cahiers du monde russe [En ligne], 49/4 | 2008, mis en ligne le 11 février 2010, consulté le 03 septembre 2022. URL : http:// journals.openedition.org/monderusse/7009; DOI : https://doi.org/10.4000/monderusse.7009

Ce document a été généré automatiquement le 3 septembre 2022

Tous droits réservés 


\title{
Ray Brandon, Wendy Lower, eds, The Shoah in Ukraine
}

\author{
Nathalie Moine
}

\section{RÉFÉRENCE}

Ray BRANDON, Wendy LOWER, eds, The Shoah in Ukraine. History, Testimony,

Memorialization. Bloomington : Indiana University Press, 2008, 378 p.

1 Le recueil d'articles conçu par Ray Brandon et Wendy Lower constitue un panorama remarquable de la recherche actuelle sur la Shoah à l'Est. Le découpage géographique, qui prend en compte les frontières actuelles de l'Ukraine, peut surprendre le spécialiste en histoire soviétique - nous y reviendrons - puisque, précisément, ni l'Ukraine actuelle ni la république socialiste soviétique d'Ukraine ne constituèrent un cadre homogène d'occupation pour les nazis. L'introduction souligne d'emblée qu'on ne saurait, non plus, parler des juifs ukrainiens comme d'une entité, alors qu'ils se répartissent entre des communautés très diverses : communautés de Galicie et de Volhynie, inscrites dans une aire culturelle germanisée puis soumises à la polonisation de l'entre-deux-guerres, tout juste confrontées à l'idéologie bolchevique à la suite de l'annexion de la Pologne orientale en septembre 1939; celles de Bessarabie et de Bucovine; et enfin les populations juives des grandes villes soviétiques d'Ukraine centrale et orientale. Ce parti pris présente cependant l'intérêt de juxtaposer et de comparer le déroulement des massacres dans des contextes d'occupation variables sur le plan institutionnel. Les contributions portent donc sur la Galicie, rattachée au Gouvernement Général, sur les provinces incluses dans le commissariat du Reich pour l'Ukraine (RKU) après une période d'administration par la Wehrmacht au cours des deux mois d'invasion, sur celles situées plus à l'Est qui restèrent sous administration militaire, enfin sur la Transnistrie, placée sous domination roumaine à partir d'août 1941. 
Ces distinctions institutionnelles jouent de fait un rôle essentiel dans l'argumentation de cet ensemble de recherches principalement centré sur la question du poids des responsabilités entre les différents acteurs du massacre des juifs.

Quels furent les principaux responsables au sein des institutions allemandes, quelles furent les institutions les plus meurtrières? Les contributions font le point sur ces questions débattues depuis de longues années au sein des historiens du nazisme. Le rôle de la Wehrmacht est ainsi réévalué aux côtés de celui des SS et des diverses forces de police, mais aussi en comparaison avec les massacres commis dans les zones soumises à une administration civile allemande. Il n'est pas question pour autant d'absoudre cette dernière. Le rôle des collaborations locales est également mis en valeur. D'où l'importance d'un récit précis dans la chronologie, le déroulement et la comptabilité des victimes, comme le montre la remarquable synthèse proposée par Dieter Pohl qui compare le massacre des juifs avant et après l'instauration de l'administration civile du commissariat du Reich pour l'Ukraine, mettant ainsi en évidence le rôle de la Wehrmacht, ainsi que de la police régulière, dans l'anéantissement des juifs sous sa domination. De même, la contribution d'Alexandre Kruglov, la seule à porter sur l'ensemble du territoire de l'Ukraine actuelle, met en valeur les différences régionales aussi bien dans le calendrier, dans le nombre, que dans la part des juifs dans le nombre total des victimes.

4 Au total, ces contributions convergent vers la mise en lumière d'une forte collusion entre les différentes institutions: si toutes ne jouaient pas toujours un rôle moteur dans la décision de conduire un massacre, elles y participèrent toutes au moins sous la forme d'un accord tacite, le plus souvent par une aide logistique plus ou moins substantielle. À cet égard, le massacre de Kamenec-Podolskij - 23000 victimes juives en deux jours dans une ville sous administration de la Wehrmacht - est considéré comme un tournant dans le massacre des juifs à grande échelle. Lorsque la Wehrmacht transfère son autorité à l'administration civile, beaucoup de juifs ont déjà été assassinés. Koch attend quelques mois avant de reprendre le massacre à grande échelle, du printemps à décembre 1942. Plusieurs contributions montrent également, au niveau local, des commandeurs de la Wehrmacht ou des commissaires de district, étudiés par Wendy Lower pour le commissarit général de Žytomyr, qui font appel aux forces de police pour les "débarrasser de la Question juive» et se trouvent physiquement présents lors des liquidations. À l'inverse, l'étude d'Andrej Angrick sur la construction de la Voie IV (Durchgangstrasse IV), route qui devait être déterminante pour l'acheminement des troupes et du ravitaillement en matériel de la Galicie à la mer Noire - surnommée « la route des SS »- montre que les responsables SS se heurtèrent en permanence à un problème de main-d'œuvre qui les mettait à l'affût des juifs rescapés des massacres, de moins en moins nombreux au fur et à mesure qu'on progressait vers l'Est, et qu'ils tentaient de récupérer pour peupler leurs camps de travail forcé. Il s'agissait de juifs en provenance de Galicie ou recrutés en Transnistrie. Un autre thème fondamental de l'historiographie du nazisme apparait ici, celui de la compétition entre institutions, aussi bien en ce qui concerne le massacre que l'appropriation de main-d'œuvre forcée, étant entendu que la fureur raciale des nazis conduisait en toutes circonstances au massacre final : les quelques juifs sélectionnés au moment de la liquidation des ghettos pour travailler sur les chantiers mouraient bientôt dans les camps, de faim, de maladie, abattus individuellement au quotidien ou collectivement à l'achèvement d'un chantier. 
Les sources utilisées pour reconstituer les faits sont ici presque exclusivement allemandes (et roumaines pour la Transnistrie), incluant les enquêtes menées dans le cadre des procès allemands d'après-guerre. Les sources soviétiques en revanche sont peu mobilisées. Alexandre Kruglov aborde la question de la fiabilité des données collectées par la Commission Extraordinaire d'enquête des Soviétiques, mais sans fournir ici les éléments d'une réelle discussion.

6 Autre parti pris méthodologique d'importance, ce premier ensemble de contributions est centré en quasi-totalité sur la période proprement dite de la Shoah, bien que Wendy Lower donne des éléments sur les origines sociales et la formation des commissaires de district qui sévirent dans la région de Žytomyr, ces promus devenant les bureaucrates de l'ordre nazi, et que l'ensemble des conclusions rappelle la faiblesse des condamnations après-guerre à l'égard de ces hommes qui réussirent à minimiser leurs responsabilités dans le génocide.

7 Les contributions portant sur des groupes spécifiques de la population locale proposent un éclairage historique plus large. L'Ukraine occidentale est ici largement présente, puisqu'elle fait l'objet de l'article de Timothy Snyder consacré aux juifs de Volhynie occidentale et de celui de Franck Golczewski sur les nationalistes ukrainiens de Galicie. Snyder donne une épaisseur historique et sociale aux victimes, rappelant l'ancienneté de leur implantation et l'importance sociale et économique d'une population vivant plutôt en bonne intelligence avec les autorités de l'empire austro-hongrois. L'entre-deuxguerres au sein du nouvel État polonais est décrit en des termes nuancés puisque, jusqu'au durcissement consécutif au décès de Pilsudski, les juifs purent tirer profit des aléas politiques et développer une vie communautaire tout en gouvernant les conseils municipaux dans les villes de Pologne orientale où ils restaient largement majoritaires. À l'inverse, la période de soviétisation, après l'annexion de septembre 1939, sonne le glas de la vie culturelle juive et affaiblit aussi considérablement les communautés sur un plan social et économique à la suite des déportations de masse dans lesquelles les juifs sont surreprésentés et de la fin du commerce privé. Cependant, ce portrait des juifs de Volhynie est moins axé sur une description de cette vie juive autonome que sur la question des relations interethniques de longue durée dans cette région, impliquant, aux côtés des juifs, les Polonais et les Ukrainiens. Les massacres interethniques de l'année 1943 en constituent le dénouement sanglant, nationalistes ukrainiens et partisans soviétiques nouvellement arrivés dans la région se retrouvant en compétition pour son contrôle face aux Allemands, au détriment des juifs, ignorés par les Soviétiques et victimes du nationalisme antisémite intégral des Ukrainiens ainsi que des Polonais. Snyder cite plusieurs cas de solidarité entre juifs et Polonais dans la région. Le fait que ces exemples soient issus de récits recueillis dans le cadre de l'Institut polonais d'histoire juive - important centre créé dans la Pologne de l'immédiat après-guerre - n'est pas commenté. L'homogénéisation ethnique de la région est parachevée par les échanges de populations au sortir de la guerre. C'est bien aussi la question des relations interethniques qui structure l'étude de Golczewski sur la région voisine de Galicie. Les protagonistes sont les mêmes : juifs, Polonais, Ukrainiens, puis Soviétiques et Allemands, mais les configurations politiques diffèrent et l'auteur souligne ainsi la différence de statut pendant l'occupation nazie entre les Ukrainiens du Gouvernement Général et ceux qui se trouvaient sous administration de la RKU ainsi que plus à l'Est en zone militaire. Alors que ces derniers sont traités en esclaves par les occupants, les Ukrainiens de Galicie, du moins les élites, bénéficient d'une bienveillance toute relative de l'occupant 
qui cherche à les utiliser dans son projet de destruction de la nation polonaise. Les privilèges grappillés, sur fond d'une haine des juifs et des démocraties occidentales, retracée par Golczewski depuis la Première Guerre mondiale, peuvent ainsi expliquer l'entêtement d'une partie des nationalistes ukrainiens dans le bien-fondé de la collaboration avec les Allemands pour ériger un hypothétique État ukrainien, tandis que les banderistes décident de prendre le maquis.

Cependant, l'immense majorité des Ukrainiens, ceux des provinces occidentales et surtout ceux des régions centrales et orientales de l'Ukraine, sont très en retrait dans l'ouvrage. L'apport conséquent des Ukrainiens à l'appareil administratif d'occupation au niveau local est souligné à plusieurs reprises, notamment du fait de l'importance des polices locales dans la réalisation des massacres contre les juifs. Le spécialiste de cette question, Martin Dean, éclaire un autre aspect de cette collaboration indigène en montrant la surreprésentation des Volksdeutsche dans les forces de répression et l'administration locale, notamment dans les postes de responsabilités, y compris dans des zones dépourvues de colonies allemandes. Ils sont également très recherchés comme traducteurs. La plupart des hommes adultes citoyens soviétiques d'origine allemande ayant été déportés plus à l'Est par les Soviétiques, ce sont bien souvent des hommes extrêmement jeunes qui se trouvèrent enrôlés par l'occupant, ou au contraire des hommes d'âge mûr à qui l'occupant confie en priorité des fonctions dans l'administration de la population. À l'autre extrémité du spectre des comportements sous occupation, les cas de sauvetage de juifs par des Ukrainiens sont rapportés, à l'aide de la documentation accumulée par Yad Vashem pour distinguer les "Justes». Cependant, l'hostilité des populations non juives est bien ce qui domine. Elle explique pourquoi l'évasion hors du ghetto pour une poignée de juifs s'avéra finalement la partie la moins terrible dans leur tentative de survie, selon la formule de Snyder, car elle s'achevait bien souvent par la dénonciation ou la mort dans les bois, faute d'aide aussi bien des habitants que des partisans soviétiques, lorsqu'il y en avait.

Néanmoins, la complexité d'une occupation où les Ukrainiens furent à la fois bourreaux et victimes n'est jamais abordée de front. La contribution d'Angrick sur les camps de travail forcé le long du chantier de la DG IV évoque bien la coexistence de prisonniers de guerre soviétiques, de civils, essentiellement paysans, ukrainiens et de juifs, mais il n'aborde pas la comparaison dans le traitement de ces travailleurs forcés.

De même, les données présentées par Kruglov évoquent la part très variable, même si elle est massivement surreprésentée, de la population juive dans l'ensemble des victimes civiles de l'occupation. Elle constitue presque $100 \%$ des victimes en Ukraine occidentale, mais $20 \%$ dans les régions de Kiev ou Dnepropetrovsk, pour les raisons suivantes: évacuation d'une partie de la population urbaine notamment juive, part de la population juive de toute façon beaucoup plus réduite qu'à l'Ouest. Notons toutefois que la comparaison dans les formes de mise à mort des civils ukrainiens et juifs n'est pas traitée.

Ce point apparaît pourtant essentiel, notamment si on prend en considération la dimension mémorielle de la Shoah à l'Est. On sait que pendant des décennies, à commencer par les années de guerre, le gouvernement soviétique mena une politique d'occultation de la spécificité de la Shoah. Le fait de placer cette dernière au cœur d'études savantes qui documentent et détaillent le génocide juif contribue de façon salutaire à affermir les connaissances sur une histoire encore opaque en bien des points, et largement refoulée. L'absence de référence au martyre ukrainien rend pourtant 
difficile le dialogue avec une opinion publique ukrainienne. La question de la mémoire de la Shoah est d'ailleurs une piste importante ouverte par l'ouvrage. La contribution de Karel Berkhoff sur les divers témoignages fournis par Dina Pronicheva, une des rares survivantes de Baby Yar, permet de complexifier l'approche de la mémoire de la Shoah à l'époque soviétique: si le discours officiel ne parle pas des victimes juives, différentes instances soviétiques ont cependant documenté spécifiquement la Shoah. Dina Pronicheva témoigne ainsi publiquement pour la première fois lors du procès de Kiev de janvier 1946, après avoir été interviewée quelques jours plus tôt par la Pravda d'Ukraine. Elle est ensuite interrogée par une commission d'historiens quelques mois plus tard et Berkhoff établit que ce témoignage de 1946 s'avère déjà très complet et servira de canevas au Babij Jar de Anatolij Kuznecov. Berkhoff retrace la publication partielle de ce roman-document dans l'Union Soviétique brejnévienne où la commémoration de Baby Yar est devenue un enjeu important pour les représentants de la communauté juive. Le témoignage majeur est cependant celui de 1968, lorsque Pronicheva est autorisée par les autorités soviétiques à venir témoigner devant un tribunal occidental, à Darmstadt. De fait, l'importante collecte d'informations menée par les Soviétiques sur les crimes de l'occupant, pour problématique qu'elle soit, contribue au renouvellement actuel d'une historiographie de la Shoah davantage axée sur les populations locales, et en premier lieu juives, ce qui constitue un point de vue absent du volume. La question des survivants, celle de la renaissance des communautés juives dans l'Ukraine d'aprèsguerre $^{1}$ ou encore des règlements de compte de l'épuration à l'époque soviétique ${ }^{2}$, n'est ainsi pas traitée dans l'ouvrage.

La contribution extrêmement percutante et hautement problématique d'Omer Bartov nous fait faire un bond dans l'Ukraine contemporaine. Elle montre de façon poignante le terrible silence qui entoure le massacre des juifs dans des villes où ils constituaient pourtant une part importante, voire majoritaire, de la population au moment de l'invasion nazie, l'éradication de la mémoire juive (mais aussi, bien qu'évoquée de façon très ténue, polonaise) menée par les autorités locales après-guerre. Plus récente, l'hégémonie mémorielle ukrainienne est synthétisée par l'image particulièrement forte des photos de combattants ukrainiens en uniforme allemand, héroïsés dans un musée abrité dans l'ancienne demeure du rabbin de la ville de Kosiv. Cependant, plusieurs questions restent en suspens, ce qui interdit de généraliser le réquisitoire mené par Omer Bartov. L'absence de recours aux méthodes de l'historien rend imprécises et donc sujettes à caution, au moins pour la périodisation, les informations concernant la destruction par les Soviétiques des quelques synagogues rescapées de l'occupation. Surtout, le voyage auquel nous convie Bartov se situe en Galicie, un des principaux foyers juifs avant-guerre, et par ailleurs foyer du nationalisme ukrainien. Il est donc délicat d'extrapoler à l'ensemble de l'Ukraine actuelle les observations faites en Galicie, même si l'occultation de la présence des juifs avant la Shoah et le silence sur leur anéantissement est sans doute à déplorer dans tout le pays. Les notations de Bartov concernant les plaques commémoratives et les monuments marquant les sites de la Shoah, sont intéressantes à plus d'un titre. Elles montrent en particulier que même les organisations juives ayant tenté de promouvoir une politique commémorative après 1991 ont intériorisé le tabou de la Shoah, omettant de préciser le mot "juif» dans le texte ukrainien. Cependant, la présence d'une étoile de David, ainsi que celle d'un texte en hébreu, ne laissent aucune ambiguïté sur l'identité des victimes. Fait cependant défaut une claire analyse de ces gestes commémoratifs avant et après 1991, sans parler de l'éventuelle césure en ce domaine opérée par la révolution orange de l'automne 2004. 
13 Ainsi, beaucoup des conclusions de l'ouvrage n'apparaîtront pas comme des nouveautés, les auteurs de différentes contributions ayant publié des monographies complètes depuis le déroulement $d u$ workshop initial qui est à l'origine du recueil. Il n'empêche que l'ouvrage réunit la synthèse des travaux des meilleurs spécialistes, éditée avec le plus grand soin, ce qui en fait un ouvrage de référence indispensable.

\section{NOTES}

1. Yaacov Ro'i, «The Reconstruction of Jewish Communities in the USSR, 1944-1947 ", in David Bankier, ed., The Jews Are Coming Back. The Return of the Jews to their Countries of Origine after WWII, New York: Berghahn Books/Yad Vashem: Jerusalem, 2005,p. 186-205 ; Mihail Micel', Obščiny judejskogo veroispovedanjia $v$ Ukraine (Kiev, L'vov: 1945-1981gg.) [Les communautés de confession juive en Ukraine (Kiev, L'vov : 1945-1981)], Kiev, 1998.

2. Tanja Penter, «Collaboration on Trial : New Source Material on Soviet Postwar Trials against Collaborators ", Slavic Review, 64 (4), Winter 2005, p. 782- 785 ; Id., « Local Collaborators on Trial: Soviet War Crimes Trials under Stalin (1943-1953) », Cahiers du Monde Russe, 49 (2-3), avrilseptembre 2008, p. 341-364. 